\title{
Video Article \\ A Highly Reproducible and Straightforward Method to Perform In Vivo Ocular Enucleation in the Mouse after Eye Opening
}

\author{
Jeroen Aerts ${ }^{* 1}$, Julie Nys ${ }^{* 1}$, Lutgarde Arckens ${ }^{1}$ \\ ${ }^{1}$ Department of Biology, KU Leuven - University of Leuven \\ * These authors contributed equally
}

Correspondence to: Lutgarde Arckens at lut.arckens@bio.kuleuven.be

URL: https://www.jove.com/video/51936

DOI: doi:10.3791/51936

Keywords: Anatomy, Issue 92, Deprivation, visual system, eye, optic nerve, rodent, mouse, neuroplasticity, neuroscience

Date Published: 10/6/2014

Citation: Aerts, J., Nys, J., Arckens, L. A Highly Reproducible and Straightforward Method to Perform In Vivo Ocular Enucleation in the Mouse after Eye Opening. J. Vis. Exp. (92), e51936, doi:10.3791/51936 (2014).

\section{Abstract}

Enucleation or the surgical removal of an eye can generally be considered as a model for nerve deafferentation. It provides a valuable tool to study the different aspects of visual, cross-modal and developmental plasticity along the mammalian visual system ${ }^{1-4}$.

Here, we demonstrate an elegant and straightforward technique for the removal of one or both eyes in the mouse, which is validated in mice of 20 days old up to adults. Briefly, a disinfected curved forceps is used to clamp the optic nerve behind the eye. Subsequently, circular movements are performed to constrict the optic nerve and remove the eyeball. The advantages of this technique are high reproducibility, minimal to no bleeding, rapid post-operative recovery and a very low learning threshold for the experimenter. Hence, a large amount of animals can be manipulated and processed with minimal amount of effort. The nature of the technique may induce slight damage to the retina during the procedure. This side effect makes this method less suitable as compared to Mahajan et al. $(2011)^{5}$ if the goal is to collect and analyze retinal tissue. Also, our method is limited to post-eye opening ages (mouse: P10 - 13 onwards) since the eyeball needs to be displaced from the socket without removing the eyelids. The in vivo enucleation technique described in this manuscript has recently been successfully applied with minor modifications in rats and appears useful to study the afferent visual pathway of rodents in general.

\section{Video Link}

The video component of this article can be found at https://www.jove.com/video/51936/

\section{Introduction}

Removing an eye and thereby irreversibly destructing the sensory receptor surface (retina), imposes a considerable loss of sensory input along the visual pathway. The enucleation model in the juvenile and adult visual system has proven to be valuable in understanding the development plasticity and function of different visual centers ${ }^{1-4}$. The molecular, cellular and physiological consequences of this sensory deprivation can provide insights into how normal development is regulated and how established cortical circuits cope and change their structure and function in response to such an extensive alteration in experience.

Different methods of visual deprivation exist and they all have their specific advantages in vision-related research. For example dark rearing specifically eliminates visually driven activity yet it does not affect the spontaneous retinal activity. Similarly, lid sutures or eye patches remove patterned visual input without disturbing spontaneous activity but they allow dispersed light penetration through the closed eyes. Those methods are reversible and have been shown to be valuable in understanding the role of patterned vision and low-level correlation of binocular inputs in sculpting cortical circuits during development ${ }^{6-8}$. In glaucoma research, the optic nerve crush model in adult animals has been widely used because it establishes a progressive loss of retinal ganglion cell inputs that constitute the optic nerve ${ }^{9,10}$. On the other hand, enucleation, where the eye and thus the retina is completely and instantly removed, is the appropriate choice of deprivation when the aim is to irreversibly remove both spontaneous and patterned vision at once. It also induces a robust intraocular activity imbalance which can enhance the signal to noise ratio in activity mapping studies ${ }^{11,12}$. Comparing functional and structural changes in response to enucleation with those after deprivation by less drastic methods such as lid suture for example, might also expose new insights into the role of spontaneous retinal activity in both homeostatic and synaptic types of plasticity.

Enucleation triggers a loss of trophic influences in direct retinal targets. For instance, BDNF levels are significantly downregulated in the lateral geniculate nucleus (LGN) and superior colliculus of adult enucleated rats ${ }^{13}$. Reactive oxygen species, which function as messenger molecules to mediate structural remodeling, were also detected in subcortical structures of the adult rat visual system ${ }^{14}$. Furthermore, microglial and astroglial activation across different subcortical visual target structures in the mouse occur in a specific post-enucleation time frame of one week ${ }^{15}$. Together, optic deafferentation results in different subcortical responses at the glial, structural and molecular level. Despite these subcortical effects, it does not necessarily implicate effects at the cortical level ${ }^{16}$. Noteworthy, cross-modal cortical plasticity, including modifications in other 
sensory areas next to the strengthening of non-visual inputs to the deprived visual cortex occur after both monocular (ME) $)^{3,4,17,18}$ and binocular $(\mathrm{BE})^{1,17}$ enucleation.

Apart from contributing to visual neuroscience, enucleation as a type of deafferentation can be used to study the balance between neuroprotective ${ }^{19}$ and neurodegenerative $\mathrm{e}^{20-22}$ properties of the central nervous system.

Different procedures to perform enucleation are already described in literature. Certain methodologies for in vivo ME in rats and mice are less straightforward due to unnecessary sectioning of orbital muscles and tissue ${ }^{23-25}$. Other publications such as Mahajan et al. (2011) ${ }^{5}$ provide a detailed protocol using blunt dissection for a high-throughput collection of eyes to study the genotype-phenotype correlations, likely post-mortem. For their purpose, the method is convenient and fast. However, this method is less suitable for in vivo enucleation when one opts to study the afferent visual pathway following enucleation (in live animals) rather than the eye itself. In such a setting, post-enucleation survival is of high importance. Also, minimal in vivo damage and preservation of the optic nerve and orbital tissue is favorable. Here, we present an alternative enucleation method, more similar to the one described by Faguet et al. $(2008)^{26}$, that offers certain advantageous properties: it is associated with a swift post-operative recovery and is characterized by a very low learning threshold for researchers. In general, different methods are complementary depending on the focus of subsequent research: eye morphology or visual pathway research.

In sum, enucleation can be applied from vision research towards investigations of homeostatic and cross-modal brain plasticity, glial response properties, and axon stability. In this visualized article, we demonstrate a feasible and reliable method for in vivo eye enucleation in the mouse.

Protocol

All experiments were approved by the ethical research committee of KU Leuven and were in strict accordance with the European Communities Council Directive of 22 September 2010 (2010/63/EU) and with the Belgian legislation (KB of 29 May 2013). Every possible effort was made to minimize animal suffering and to reduce the number of animals.

\section{Animal Treatment and Anesthetics}

1. Anesthetize the mouse with an intraperitoneal injection of a mixture of ketamine hydrochloride ( $75 \mathrm{mg} / \mathrm{ml})$ and medetomidine hydrochloride ( 1 $\mathrm{mg} / \mathrm{kg}$ ) in saline.

2. Check the reflexes by pinching the toes with a forceps to assure the mouse is completely sedated.

3. Apply $70 \%$ ethanol to disinfect the eyelids and the region surrounding the eye using a cotton tip. Check the eyelid reflex to additionally assess the degree of sedation.

\section{Removing the Eye}

1. Make sure the animal resides on a flat, dry and smooth surface.

2. Sterilize a forceps with a curved, serrated tip (preferred tip size: $0.5 \times 0.4 \mathrm{~mm}$ ).

3. Gently press onto the canthus (corner of the eye) with the forceps until the eyeball is displaced from the socket and the optic nerve is reachable.

4. Guide the forceps behind the eye. Press and hold the optic nerve firmly, preferably with the beginning of the curve and not the very tip of the forceps. This will help to lift the globe from the socket and to clamp the complete optic nerve.

5. Make circular movements with the hand holding the forceps in the direction with the least resistance while the mouse remains on the flat surface. The mouse will swing along the surface according to the direction of the hand movement.

6. Perform this action with gradually increasing speed until the optic nerve is constricted in two (usually between 7 to 15 circular movements, approximately a half to one full turn per second). Hence, the detached eyeball is removed.

\section{Post-operative Care}

1. In case of bleeding (rare), fill the orbit with a viscous coagulate and hemostatic agent.

2. Reverse the anesthesia by injecting $1 \mathrm{mg} / \mathrm{kg}$ of atipamezol hydrochloride in saline intraperitoneally.

3. Administer $1 \mathrm{mg} / \mathrm{kg}$ of Meloxicam intraperitoneally every $24 \mathrm{hr}$ to relieve pain.

4. Apply eye ointment to the remaining eye to prevent dehydration of the cornea.

5. Let the animal recover on a heating plate or wrap the animal in insulating material in a separate cage to control body temperature.

6. Measure the weight of the mouse each day for at least 2 days. Loss of weight can indicate suffering and in this case, continue Meloxicam treatment until the animal is fully recovered.

\section{Representative Results}

Figure 1 illustrates the successful removal of the eye using the described protocol and is characterized by the absence of bleeding or any apparent physical damage to the orbital tissue or eye socket (Figures 1A, 1B). Furthermore, the removed eye has a smooth cornea, choroid and optical disc, indicative for a completely intact globe (Figure 1C). Since our protocol encompasses the clamping of the optic nerve behind the eye and mechanical turning, the optic nerve of the removed eye is constricted at the base of the retina (Figure 1D). Performing the described procedure results in a clean-cut optic nerve without any damage to the surrounding brain area (Figure 1E).

Monocular enucleation, in combination with activity mapping (Figure 2), allows one to sharply delineate the functional or eye specific input regions in the contralateral visual cortex of the mouse ${ }^{12,27}$ or even ocular dominance columns in higher-order mammals like monkeys ${ }^{28}$. 
In experiments with mice, the removal of one (ME) or both eyes (BE) combined with targeted visual stimulation and the detection of zif268 mRNA or C-Fos protein expression levels was applied to uncover regional neuronal activation in the visual cortex ${ }^{12,27}$. In contrast to visually stimulated controls (Figure 2A), BE mice showed basal activity in the visual cortex due to complete lack of visual input (Figure 2B). As such, the borders between visual with non-visual cortex (i.e. somatosensory cortex at more anterior sections and auditory cortex in more posterior sections) were uncovered. Results from ME mice with a one-week survival time visualized the eye specific input regions in the contralateral visual cortex. The two monocularly driven regions were hypoactive and located medial and lateral of the central binocular zone (Figure 2C).
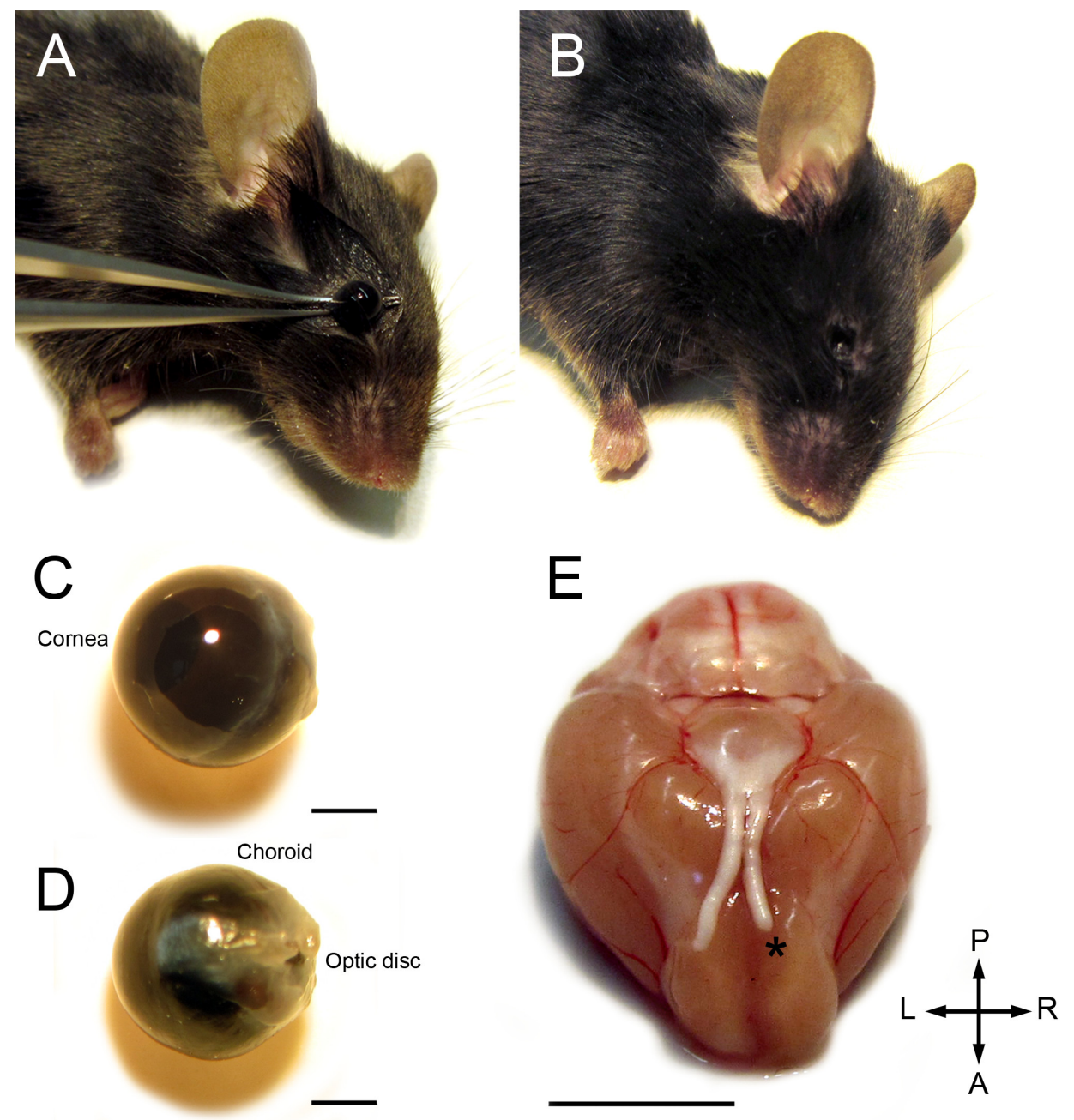

Figure 1. Qualitative evaluation of the post-enucleation state of the eye socket, the removed eye and the optic nerve. After removal of the eye with a curved forceps $(\mathbf{A})$ no bleeding or damage is observed in the eye socket (B). The removed eye is completely intact as reflected by a normal appearance of the cornea and choroid (C,D). The optic nerve is constricted at the optic disc where it leaves the eye (D). Examination of the ventral part of the brain reveals a clean cut optic nerve (asterisk) and no apparent damage to other structures (E). Scale bars in C, D: $1 \mathrm{~mm}$. Scale bar in E: 5 mm. A: anterior; L: left; P: posterior; R: right. 

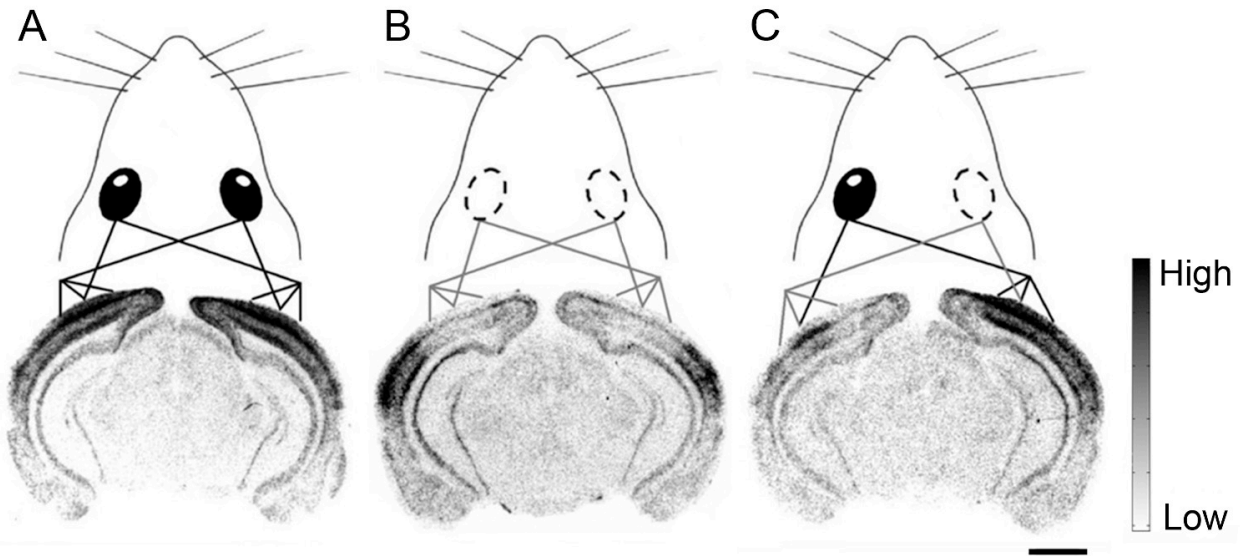

Figure 2. Functional eye-input specific subdivisions in the mouse visual cortex as revealed by enucleation. Black and gray lines that connect the eyes and cortex represent the crossing over of retinal afferents and the eye specific input regions. Neural activity is visualized on coronal sections of control (A), BE (B), and ME (C) mice by radioactive in situ hybridization (ISH) for zif268 (gray scale) around Bregma level $-3.40 \mathrm{~mm}$. In control animals (A), the visual cortex in both hemispheres expresses high activity following visual stimulation. When one or both eye(s) are extirpated, a clear decrease in the activity signal is visible in corresponding deprived cortical regions. Monocularly enucleated (C) mice show a zone of high activity in the binocular zone of the visual cortex surrounded by a decreased signal in the monocular zones contralateral to the removed eye. Scale bar: $2 \mathrm{~mm}$. Reprinted with permission from Van Brussel et al ${ }^{12}$.

\section{Discussion}

To perform a successful enucleation according to our method, the most critical steps to consider are: 1) using a forceps with a curved and serrated tip of the appropriate size; 2) performing the enucleation on a smooth and dry surface; and 3) gradually speeding up the circular movements in the direction with the least friction.

For an effective outcome it is essential to use an appropriate forceps characterized by a curved and serrated tip (preferred tip size: mouse: $0.5 \times 0.4 \mathrm{~mm}$; rat: $2.15 \times 1.3 \mathrm{~mm}$ ). The curvature allows for an easy access to the optic nerve after eyeball displacement and is necessary for correct hand placement when performing the circular movements. Smooth tips are not recommended since they lack the necessary grip when holding the optic nerve. Failure to hold the optic nerve properly during the circular movements results in rupture of the ophthalmic artery, poor detachment of the eye and hence poor reproducibility. Therefore it is recommended to first practice this technique on euthanized animals for optimization of the forceps handling in order to guarantee maximal animal welfare once applying the method in vivo. Successful eye enucleation has recently also been performed in the rat in our lab using the same technique except for turning the animal body manually and keeping the forceps stationary.

One limitation of the technique is that it could possibly damage the retina. Therefore this method is less suitable for collecting retinas to perform histology ${ }^{5}$. Moreover, our method is limited to post eye opening ages since the eyeball needs to be displaced from the socket without removing or cutting the eyelids.

Eye enucleation in different species, including rodents, is routinely performed using alternative methods, which often entail the removal of the eyelids and cutting the optic nerve ${ }^{18,23-25}$. These methods tend to be more invasive and have a higher learning curve than the technique described here. Without the need for removing or suturing the eyelids, the post surgery recovery time is minimized, resulting in higher animal welfare and more reproducible results.

\section{Disclosures}

The authors have nothing to disclose.

\section{Acknowledgements}

We thank Ellen Ytebrouck for her valuable information regarding method application in rat. This work was supported by the Research Council of the KU Leuven (OT09/22) and the Fund for Scientific Research-Flanders (FWO Vlaanderen). Jeroen Aerts is supported by a Ph.D. fellowship of the Agency for Innovation through Science and Technology Flanders (IWT Vlaanderen) and Julie Nys by a Ph.D. grant from FWO Flanders.

\section{References}

1. Toldi, J., Fehér, O., \& Wolff, J. R. Neuronal plasticity induced by neonatal monocular (and binocular) enucleation. Progress in Neurobiology. 48 (3), 191-218, (1996).

2. Steeves, J. K. E., González, E. G., \& Steinbach, M. J. Vision with one eye: a review of visual function following unilateral enucleation. Spatial vision. 21 (6), 509-529, (2008).

3. Van Brussel, L., Gerits, A., \& Arckens, L. Evidence for cross-modal plasticity in adult mouse visual cortex following monocular enucleation. Cerebral Cortex. 21 (9), 2133-2146, (2011). 
4. Nys, J., Aerts, J., Ytebrouck, E., Vreysen, S., Laeremans, A., \& Arckens, L. The cross-modal aspect of mouse visual cortex plasticity induced by monocular enucleation is age-dependent. Journal of Comparative Neurology. 522 (4), 950-970, (2014).

5. Mahajan, V. B., Skeie, J. M., Assefnia, A. H., Mahajan, M., \& Tsang, S. H. Mouse eye enucleation for remote high-throughput phenotyping Journal of Visualized Experiments. (57), doi:10.3791/3184, (2011).

6. Morales, B., Choi, S.-Y., \& Kirkwood, A. Dark rearing alters the development of GABAergic transmission in visual cortex. Journal of Neuroscience. 22 (18), 8084-8090, (2002).

7. Chen, X. J., Rasch, M. J., Chen, G., Ye, C. Q., Wu, S., \& Zhang, X. H. Binocular input coincidence mediates critical period plasticity in the mouse primary visual cortex. Journal of Neuroscience. 34 (8), 2940-2955, (2014).

8. Konur, S., \& Yuste, R. Developmental regulation of spine and filopodial motility in primary visual cortex: Reduced effects of activity and sensory deprivation. Journal of Neurobiology. 59 (2), 236-246, (2004).

9. Parrilla-Reverter, G. et al. Time-course of the retinal nerve fibre layer degeneration after complete intra-orbital optic nerve transection or crush: A comparative study. Vision Research. 49 (23), 2808-2825, (2009).

10. Galindo-Romero, C. et al. Axotomy-induced retinal ganglion cell death in adult mice: Quantitative and topographic time course analyses. Experimental Eye Research. 92 (5), 377-387, (2011).

11. Kanold, P. O., Kim, Y. A., GrandPre, T., \& Shatz, C. J. Co-regulation of ocular dominance plasticity and NMDA receptor subunit expression in glutamic acid decarboxylase-65 knock-out mice. The Journal of Physiology. 587 (12), 2857-2867, (2009).

12. Van Brussel, L., Gerits, A., \& Arckens, L. Identification and localization of functional subdivisions in the visual cortex of the adult mouse. Journal of Comparative Neurology. 514 (1), 107-116, (2009).

13. Avwenagha, O., Bird, M. M., Lieberman, A. R., Yan, Q., \& Campbell, G. Patterns of expression of brain-derived neurotrophic factor and tyrosine kinase B mRNAs and distribution and ultrastructural localization of their proteins in the visual pathway of the adult rat. Neuroscience. 140 (3), 913-928, (2006).

14. Hernandes, M. S., Britto, L. R. G., Real, C. C., Martins, D. O., \& Lopes, L. R. Reactive oxygen species and the structural remodeling of the visual system after ocular enucleation. Neuroscience. 170 (4), 1249-1260, (2010).

15. Cuyvers, A., Paulussen, M., Smolders, K., Hu, T-T., \& Arckens, L. Local cell proliferation upon enucleation in direct retinal brain targets in the visual system of the adult mouse. Journal of Experimental Neuroscience. 4, 1-15, (2010).

16. Smith, S. L., \& Trachtenberg, J. T. Experience-dependent binocular competition in the visual cortex begins at eye opening. Nature Neuroscience. 10, (3) 370-375, (2007).

17. Toldi, J., Farkas, T., \& Völgyi, B. Neonatal enucleation induces cross-modal changes in the barrel cortex of rat. A behavioural and electrophysiological study. Neuroscience Letters. 167 (1-2), 1-4, (1994).

18. Newton, J. R., Sikes, R. W., \& Skavenski, A. A. Cross-modal plasticity after monocular enucleation of the adult rabbit. Experimental Brain Research. 144 (4), 423-429, (2002).

19. Lalonde, J., \& Chaudhuri, A. Dynamic changes in CREB phosphorylation and neuroadaptive gene expression in area V1 of adult monkeys after monocular enucleation. Molecular and Cellular Neuroscience. 35 (1), 24-37, (2007).

20. You, Y., Gupta, V. K., Graham, S. L., \& Klistorner, A. Anterograde degeneration along the visual pathway after optic nerve injury. PLoS ONE. 7 (12), e52061, (2012).

21. Kelly, K. R., McKetton, L., Schneider, K. A., Gallie, B. L., \& Steeves, J. K. E. Altered anterior visual system development following early monocular enucleation. Neurolmage: Clinical. 4, 72-81 (2014).

22. Chow, A. M., Zhou, I. Y., Fan, S. J., Chan, K. W. Y., Chan, K. C., \& Wu, E. X. Metabolic changes in visual cortex of neonatal monocular enucleated rat: a proton magnetic resonance spectroscopy study. International Journal of Developmental Neuroscience. 29 (1), 25-30, (2011).

23. Dyer, R. S., \& Hammond, M. Effects of enucleation in retinal degenerate mice. Physiology \& behavior. 14 (2), 207-210, (1975).

24. Smith, S. A., \& Bedi, K. S. Unilateral eye enucleation in adult rats causes neuronal loss in the contralateral superior colliculus. Journal of Anatomy. 190 (4), 481-490, (1997).

25. Gonzalez, D. et al. Effects of monocular enucleation on calbindin-D 28k and c-Fos expression in the lateral geniculate nucleus in rats. Okajimas folia anatomica Japonica. 82 (1), 9-18, (2005).

26. Faguet, J., Maranhao, B., Smith, S. L., \& Trachtenberg, J. T. Ipsilateral eye cortical maps are uniquely sensitive to binocular plasticity. Journal of Neurophysiology. 101 (2), 855-861, (2008).

27. Van der Gucht, E., Hof, P. R., Van Brussel, L., Burnat, K., \& Arckens, L. Neurofilament protein and neuronal activity markers define regional architectonic parcellation in the mouse visual cortex. Cerebral Cortex. 17 (12), 2805-2819, (2007).

28. Chaudhuri, A., Matsubara, J. A., \& Cynader, M. S. Neuronal activity in primate visual cortex assessed by immunostaining for the transcription factor Zif268. Visual Neuroscience. 12 (1), 35-50, (1995). 